\title{
Integration of Soft X-ray Tomography and High Resolution Fluorescence Microscopy
}

$\underline{\text { Zi-Jing Lin }}^{1 *}$, Chih-Wei Chen ${ }^{2}$, Chia-Chun Hsieh ${ }^{1}$, Tsung-Wen Chen ${ }^{1}$, Duan-Jen Wang ${ }^{1}$, Su-Yu Chiang $^{1}$ and Lee-Jene Lai ${ }^{1 *}$

1. National Synchrotron Radiation Research Center, Hsinchu, Taiwan

2. Industrial Technology Research Institute, Hsinchu, Taiwan

* Corresponding author, lin.zj@nsrrc.org.tw, jene@ nsrrc.org.tw

Soft X-ray Tomography (SXT) is a relatively new synchrotron-based imaging technique for biologist to observe the ultrastructure insides cells. SXT uses the X-ray source in the so called "water window" region, which is between the K-edge absorption of carbon at $284 \mathrm{eV}$ and K-edge absorption of oxygen at $543 \mathrm{eV}$. At these region, the absorption coefficient of oxygen (i.e. water) is much lower than carbon and nitrogen, which are the major biochemical constituents of biomolecules. Given this fact, SXT is able to produce high natural contrasts between cells and water as cells were only frozen without staining and processing [1]. Currently, SXT beamline and endstation are constructing at Taiwan Photon Source (TPS) in National Synchrotron Radiation Research Center (NSRRC). The SXT microscopy at TPS is designed with capillary condenser and an objective Fresnel zoneplate with spatial resolution of 15-30nm for 2D imaging and 50nm for 3D tomography.

It has been demonstrated that SXT provides morphological information of subcellular organelles at nanometer scale resolution in three dimensions (3D) [2]. However, for those organelles with similar shapes or contrast, it is difficult to identify by morphology alone. Optical fluorescence microscopy has been widely used to track the specific organelles/molecules by labeling with a fluorescent dye or expressing of the fluorescent protein. Combined SXT and optical fluorescence microscopy is capable to provide both morphological and function information inside cells for biomedical researchers [3]. As the resolution of conventional fluorescence microscopy is limited by diffraction, in order to bridge the relation of structure and function of cellular organelles more accurate, we have integrated the structured illumination fluorescence microscopy (SIFM) as the correlative fluorescence microscopy (Fig. 1). SIFM is a super resolution using a periodic light pattern to illuminate samples. This integration not only accelerates SXT imaging to target regions of interest but enables one to acquire composite 3D images to obtain rich correlation information to identify and resolve unknown intracellular structures.

The design of a correlative SIFM system based on a spatial light modulator (SLM). SLM is used to diffract laser beam into 0 and \pm 1 orders at orientations $0^{\circ}, 60^{\circ}$, and $120^{\circ}$. The system is divided into two parts - in vacuum and in atmosphere- to meet the geometry of the vacuum chamber (Figs. 2A and 2B). This system is equipped with three laser beams at 405, 488 and $532 \mathrm{~nm}$ to excite different fluorophores insides frozen cells. Achromatic lenses and waveplates have been used to correct the effects of chromatic and spherical aberration. The three diffracted beams are collected and focused with 4-lens system and then through the view port of the vacuum chamber, collected and focused with L5 and reflection mirror onto the back focal plane of an objective to intersect each other to form a three beam interference structured pattern on the sample plane. Prior to the installation to SXT endstation, SIFM system has been tested off-line using frozen florescent beads immersed in liquid nitrogen and shown nearly double resolution improvement, as shown in Fig.2D. SIFM is installing into SXT endstation and interference structured pattern have been observed from the SXT sample holder (Fig. 2C). We plan to start commissioning the SIFM with SXT microscope in the middle of 2018. 
References:

[1] C.A. Larabell and K.A. Nugent, Current Opinion in Structural Biology 20 (2010), p. 623.

[2] A.J. Pérez-Berná et al, ACS Nano 10 (2016), p. 6597.

[3] H.Y. Chen et al, Scientific Reports 6 (2016), p. 34879

[4] The authors acknowledge funding from the National Synchrotron Radiation Research Center and Ministry of Science and Technology of Taiwan under Contract No. MOST 102-3111-M-213-002

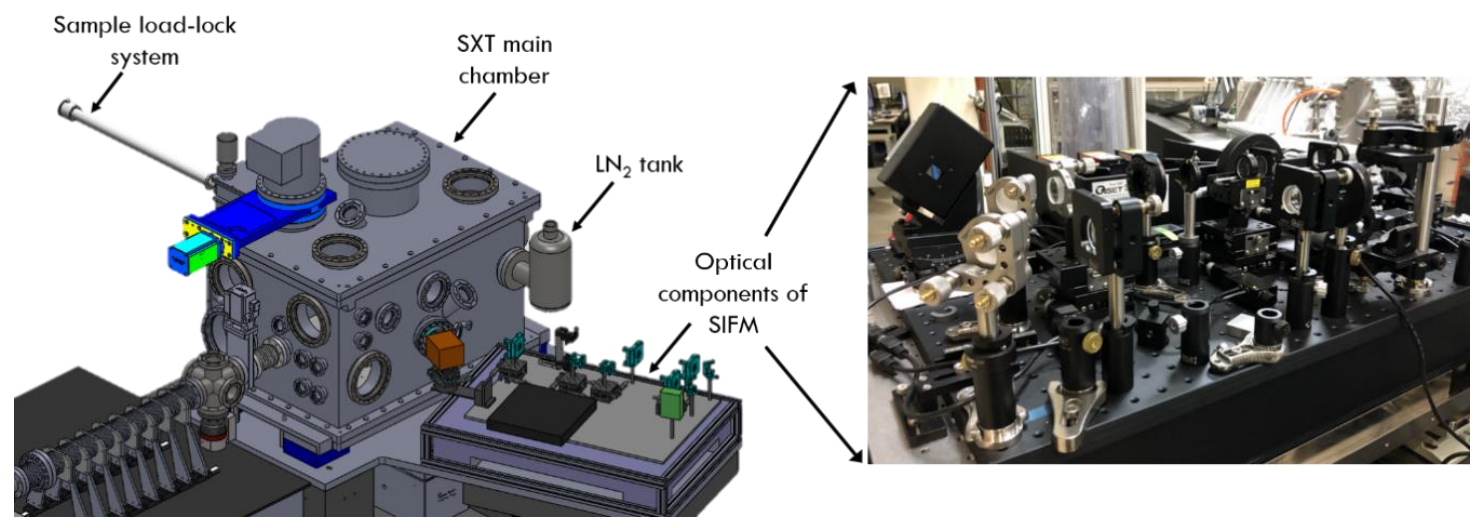

Figure 1. Schematic of soft X-ray tomography endstation. (Left) Drawing of the endstation, including the SXT microscopy and structural illumination fluorescence microscopy (SIFM). (Right) optical components of SIFM
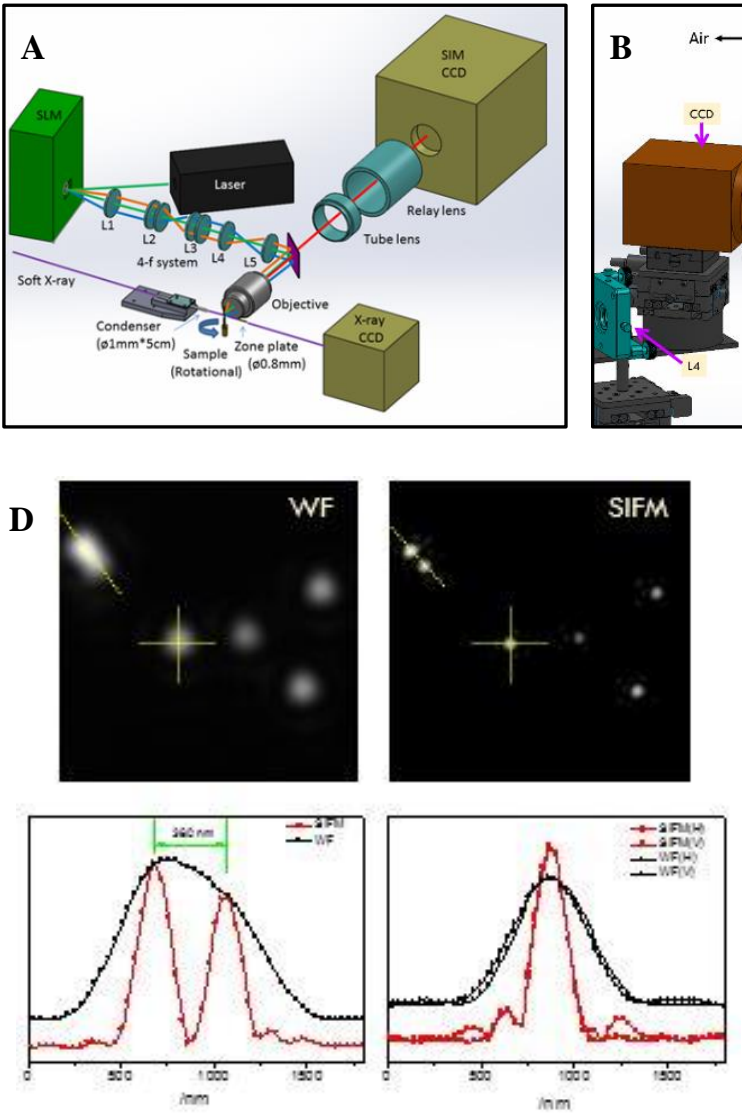

Figure 2. (A) Schematic of the integration of SXT and SIFM. (B) 3D drawing of the two parts of SIFM. (C) Three beam interference structured pattern observed from the on-line SIFM. (D)The lateral and axial resolution are 428 and $1434 \mathrm{~nm}$ in the wide filed (WF) image, and 199 and $663 \mathrm{~nm}$ in 3D-SIFM image. Improving factors of both are about 2.2. 